DOI: $10.15393 /$ j3.art.2016.3250

UDC 517.53

\author{
S. L. WALi ${ }^{1}$, W. M. SHAh ${ }^{2}$, A. Liman $^{3}$
}

\title{
INEQUALITIES CONCERNING B-OPERATORS
}

Abstract. Let $\mathcal{P}_{n}$ be the class of polynomials of degree at most $n$. Rahman introduced the class $\mathcal{B}_{n}$ of operators $B$ that map $\mathcal{P}_{n}$ into itself. In this paper we prove some results concerning such operators and thereby obtain generalizations of some well known polynomial inequalities.

Key words: polynomials, B-operator, inequalities in the complex domain, zeros

2010 Mathematical Subject Classification: 30A06, 30A64, $30 E 10$

1. Introduction. Let $\mathcal{P}_{n}$ be the class of polynomials $f(z):=\sum_{j=0}^{n} a_{j} z^{j}$ of degree at most $n$ with complex coefficients and $f^{\prime}(z)$ be their derivatives. It is well known (for reference see [1]) that

$$
\max _{|z|=1}\left|f^{\prime}(z)\right| \leq n \max _{|z|=1}|f(z)|
$$

and [2, p. 158]

$$
\max _{|z|=R>1}|f(z)| \leq R^{n} \max _{|z|=1}|f(z)| .
$$

For the class of polynomials $f \in \mathcal{P}_{n}$ satisfying $f(z) \neq 0$ in $|z|<1$, inequalities (1) and (2) have been respectively replaced by (see [3] and [4])

$$
\max _{|z|=1}\left|f^{\prime}(z)\right| \leq \frac{n}{2} \max _{|z|=1}|f(z)|
$$

and

$$
\max _{|z|=R>1}|f(z)| \leq \frac{R^{n}+1}{2} \max _{|z|=1}|f(z)| .
$$


Concerning refinements of inequalities (1) and (2) for polynomials $f(z)$ satisfying $f(0)=0$ one can refer to [5, p. 70]. Formulated a little differently, inequalities (1) and (2) say that if $f$ is a polynomial of degree at most $n$ such that $|f(z)| \leq M\left(=\left|M z^{n}\right|\right)$ for $|z|=1$, then

$$
\left|f^{\prime}(z)\right| \leq\left|\frac{d}{d z}\left(M z^{n}\right)\right|
$$

and

$$
|f(R z)| \leq\left|M R^{n} z^{n}\right|, \text { for }|z|=1 \text { and } R>1 .
$$

Inequality (5) can be seen as a special case of the following result (see $[6]$ or [7).

Theorem A. Let $F(z):=\sum_{j=0}^{n} A_{j} z^{j}$ be a polynomial of degree $n$, having all its zeros in the closed unit disk. Further, if $f(z):=\sum_{j=0}^{n} a_{j} z^{j}$ is a polynomial of degree at most $n$ such that $|f(z)| \leq|F(z)|$ for $|z|=1$, then

$$
\left|f^{\prime}(z)\right| \leq\left|F^{\prime}(z)\right|, \quad(1 \leq|z|<\infty) .
$$

Equality holds in (7) at some point outside the closed unit disk if and only if $f(z) \equiv e^{i \gamma} F(z)$ for some $\gamma \in \mathcal{R}$.

Inequality (6) follows immediately from the following result which is a special case of the Bernstein - Walsh lemma (see [8, Corollary 12.1.3]).

Theorem B. Let $F(z)$ be a polynomial of degree n, having all its zeros in the closed unit disk $|z| \leq 1$. Furthermore, let $f(z)$ be a polynomial of degree at most $n$ such that $|f(z)| \leq|F(z)|$ for $|z|=1$. Then

$$
|f(z)|<|F(z)| \text { for }|z|>1,
$$

unless $f(z) \equiv e^{i \gamma} F(z)$ for some $\gamma \in \mathcal{R}$.

2. A class of $B_{n}$-operators. Let $B$ be a linear operator carrying polynomials in $\mathcal{P}_{n}$ into polynomials in $\mathcal{P}_{n}$. We say $B$ is a $\mathcal{B}_{n}$-operator, if for every polynomial $P$ of degree $n$ having all its zeros in the closed unit disk, $B[P]$ has all its zeros in the closed unit disk.

Rahman [9] introduced this class $\mathcal{B}_{n}$ of operators $B$ and observed that if $\lambda_{0}, \lambda_{1}$ and $\lambda_{2}$ be such that

$$
\lambda_{0}+C(n, 1) \lambda_{1} z+C(n, 2) \lambda_{2} z^{2} \neq 0, \quad C(n, r)=\frac{n !}{r !(n-r) !}
$$


for $\operatorname{Re}(z) \geq \frac{n}{4}$, then the operator $B$, which associates with a polynomial $P$ of degree at most $n$ the polynomial

$$
B[P](z):=\lambda_{0} P(z)+\lambda_{1}\left(\frac{n z}{2}\right) \frac{P^{\prime}(z)}{1 !}+\lambda_{2}\left(\frac{n z}{2}\right)^{2} \frac{P^{\prime \prime}(z)}{2 !}
$$

is a $\mathcal{B}_{n}$-operator. This can also be deduced from the result of Marden [10, Corollary 18.3]. Concerning this class of operators Rahman [9] observed:

Theorem C. If $P(z)$ is a polynomial of degree $n$, then

$$
|P(z)| \leq M, \quad|z|=1,
$$

implies

$$
|B[P](z)| \leq M\left|B\left[E_{n}\right]\right|, \quad|z| \geq 1,
$$

where $E_{n}(z):=z^{n}$.

As an improvement of (9), Shah and Liman [11] proved the following:

Theorem D. If $P \in \mathcal{P}_{n}$ and $P(z) \neq 0$ in $|z|<1$, then

$$
|B[P](z)| \leq \frac{1}{2}\left(\left|B\left[E_{n}\right]\right|+\left|\lambda_{0}\right|\right) \max _{|z|=1}|P(z)|, \quad|z| \geq 1 .
$$

The result is sharp and the equality holds for a polynomial with all zeros on the unit disk.

For suitable choices of $\lambda_{0}, \lambda_{1}$ and $\lambda_{2}$ (see [11]), Theorem $\mathrm{C}$ yields inequalities (1) and (2), whereas Theorem D yields inequalities (3) and (4).

In this paper we inter alia prove a more general result which includes Theorem A and Theorem B as special cases and as such provides a combined generalization of inequalities (1) and (2). We further show that many known polynomial inequalities can also be deduced from our result. In fact, we prove the following results.

\section{Main results.}

Theorem 1. Let $f(z)$ and $F(z)$ be two polynomials of degree $m$ and $n$ $(m \leq n)$ respectively. If $F(z)$ has all its zeros in $|z| \leq 1$ and $|f(z)| \leq|F(z)|$ for $|z|=1$, then for any complex number $\beta$ with $|\beta| \leq 1$ and $R \geq r \geq 1$, we have for $|z| \geq 1$

$$
\begin{aligned}
\mid B[f](R z)-\beta B[f](r z)+\lambda_{1} \frac{n-m}{2} z\left\{(f(R z))^{\prime}-\beta(f(r z))^{\prime}\right\}+ \\
+\lambda_{2} \frac{n^{2}-m^{2}}{8} z^{2}\left\{(f(R z))^{\prime \prime}-\beta(f(r z))^{\prime \prime}\right\}|\leq| B[F](R z)-\beta B[F](r z) \mid,
\end{aligned}
$$


where $\lambda_{0}, \lambda_{1}, \lambda_{2}$ are such that if $u(z):=\lambda_{0}+C(n, 1) \lambda_{1} z+C(n, 2) \lambda_{2} z^{2}$ has all zeros in $\operatorname{Re}(z) \leq \frac{n}{4}$ then $v(z):=\lambda_{0}+C(m, 1) \lambda_{1} z+C(m, 2) \lambda_{2} z^{2}$ has all zeros in $\operatorname{Re}(z) \leq \frac{m}{4}$. The strict inequality holds in $(10)$, if $|z|>1$, unless $f(z) \equiv e^{i \gamma} F(z), \gamma \in \mathcal{R}$.

Theorem 2. If $F(z)$ and $f(z)$ are polynomials of degree $n$ such that $F(z)$ has all its zeros in $|z| \leq 1$, and $|f(z)| \leq|F(z)|$ for $|z|=1$, then for $|z| \geq 1$

$$
\left|\lambda n^{2} f(z)-z f^{\prime}(z)-z^{2} f^{\prime \prime}(z)\right| \leq\left|\lambda n^{2} F(z)-z F^{\prime}(z)-z^{2} F^{\prime \prime}(z)\right|,
$$

where $\lambda$ belongs to the set

$$
\triangle:=\left\{w=\rho+i \sigma: 0 \leq \sigma^{2} \leq \frac{n}{n-1}\left(\frac{n+1}{4 n}-\rho\right), \rho \leq \frac{n+1}{4 n}\right\} .
$$

4. Applications. The following result is an immediate application of Theorem 1 , if $m=n$.

Corollary 1. Let $f(z)$ and $F(z)$ be two polynomials of degree $n$. If $F(z)$ has all its zeros in $|z| \leq 1$ and $|f(z)| \leq|F(z)|$ for $|z|=1$, then for any complex number $\beta$ with $|\beta| \leq 1$ and $R \geq r \geq 1$, we have for $|z| \geq 1$

$$
|B[f](R z)-\beta B[f](r z)| \leq|B[F](R z)-\beta B[F](r z)| .
$$

Equivalently,

$$
\begin{aligned}
\mid \lambda_{0}\{f(R z) & -\beta f(r z)\}+\lambda_{1}\left(\frac{n z}{2}\right) \frac{R f^{\prime}(R z)-\beta r f^{\prime}(r z)}{1 !}+ \\
& +\lambda_{2}\left(\frac{n z}{2}\right)^{2} \frac{R^{2} f^{\prime \prime}(R z)-\beta r^{2} f^{\prime \prime}(r z)}{2 !} \mid \leq \\
& \leq \mid \lambda_{0}\{F(R z)-\beta F(r z)\}+\lambda_{1}\left(\frac{n z}{2}\right) \frac{R F^{\prime}(R z)-\beta r F^{\prime}(r z)}{1 !}+ \\
& +\lambda_{2}\left(\frac{n z}{2}\right)^{2} \frac{R^{2} F^{\prime \prime}(R z)-\beta r^{2} F^{\prime \prime}(r z)}{2 !} \mid \quad \text { for }|z| \geq 1 . \quad(11)
\end{aligned}
$$

The result is sharp and equality holds for the polynomial $f(z) \equiv e^{i \gamma} F(z)$, where $\gamma \in \mathcal{R}$ and $F(z)$ is any polynomial having all its zeros in $|z| \leq 1$.

If in inequality (11) we choose $\lambda_{1}=\lambda_{2}=0$, we get the result of Govil et. al [12]. Further if we choose $\lambda_{0}=\lambda_{2}=0$, we get for $R \geq r \geq 1$ and $|\beta| \leq 1$,

$$
\left|R f^{\prime}(R z)-\beta r f^{\prime}(r z)\right| \leq\left|R F^{\prime}(R z)-\beta r F^{\prime}(r z)\right| \text { for }|z| \geq 1 .
$$


Similarly for $\lambda_{0}=\lambda_{1}=0$, we have

$$
\left|R^{2} f^{\prime \prime}(R z)-\beta r^{2} f^{\prime \prime}(r z)\right| \leq\left|R^{2} F^{\prime \prime}(R z)-\beta r^{2} F^{\prime \prime}(r z)\right| \text { for }|z| \geq 1 \text {. }
$$

In case we choose $\lambda_{1}=\lambda_{2}=\beta=0$ and $R=1$ in inequality 11, we get Theorem B, whereas Theorem A easily follows from this inequality if we set $\lambda_{0}=\lambda_{2}=\beta=0, R=1$.

Remark 1. If in inequality (10) we take $\beta=r=1$ and then divide both sides by $(R-1)$, we get after making $R \rightarrow 1$, the following generalization of Theorem $A$.

Corollary 2. Let $f(z)$ and $F(z)$ be two polynomials of degree $n$. If $F(z)$ has all its zeros in $|z| \leq 1$ and $|f(z)| \leq \mid F(z)$ for $|z|=1$, then

$$
\left|B^{\prime}[f](z)\right| \leq\left|B^{\prime}[F](z)\right| \text { for }|z| \geq 1 .
$$

Equivalently

$$
\begin{aligned}
& \left|\left(\lambda_{0}+\frac{n \lambda_{1}}{2}\right) z f^{\prime}(z)+\left(\frac{n}{2} \lambda_{1}+\frac{n^{2} \lambda_{2}}{4}\right) z^{2} f^{\prime \prime}(z)+\frac{n^{2} \lambda_{2}}{8} z^{3} f^{\prime \prime \prime}(z)\right| \leq \\
& \leq\left|\left(\lambda_{0}+\frac{n \lambda_{1}}{2}\right) z F^{\prime}(z)+\left(\frac{n}{2} \lambda_{1}+\frac{n^{2} \lambda_{2}}{4}\right) z^{2} F^{\prime \prime}(z)+\frac{n^{2} \lambda_{2}}{8} z^{3} F^{\prime \prime \prime}(z)\right|
\end{aligned}
$$

for $|z| \geq 1$.

Theorem A is a special case of Corollary 2, when we take $\lambda_{1}=\lambda_{2}=0$. Setting $\lambda_{0}=\lambda_{2}=0$ and $\lambda_{0}=\lambda_{1}=0$ respectively in inequality (12), we get the following results.

Let $f(z)$ and $F(z)$ be two polynomials of degree $n$ such that $F(z)$ has all its zeros in $|z| \leq 1$ and $|f(z)| \leq|F(z)|$ for $|z|=1$; then,

$$
\left|f^{\prime}(z)+z f^{\prime \prime}(z)\right| \leq\left|F^{\prime}(z)+z F^{\prime \prime}(z)\right|
$$

and

$$
\left|f^{\prime \prime}(z)+\frac{z}{2} f^{\prime \prime \prime}(z)\right| \leq\left|F^{\prime \prime}(z)+\frac{z}{2} F^{\prime \prime \prime}(z)\right| \quad \text { for }|z| \geq 1 .
$$

In Corollary 1, if we take $F(z)=M z^{n}$, where $M=\max _{|z|=1}|f(z)|$, then we get the following: 
Corollary 3. If $f(z)$ is a polynomial of degree $n$, then for any complex number $\beta$ with $|\beta| \leq 1$ and $R \geq r \geq 1$ we have

$$
|B[f](R z)-\beta B[f](r z)| \leq\left|R^{n}-\beta r^{n}\right|\left|B\left[E_{n}\right]\right| \max _{|z|=1}|f(z)| \text { for }|z| \geq 1,
$$

where $E_{n}(z)=z^{n}$. The result is sharp and equality holds for $f(z) \equiv$ $\equiv \gamma z^{n}, \gamma \neq 0$.

If we choose $\beta=0$ in inequality (13), we get the following generalization of inequality (2).

Corollary 4. If $f(z)$ is a polynomial of degree $n$, then for $R \geq 1$ we have

$$
|B[f](R z)| \leq R^{n}\left|B\left[E_{n}\right]\right| \max _{|z|=1}|f(z)|, \text { for }|z| \geq 1 .
$$

Equivalently

$$
\begin{aligned}
& \left|\lambda_{0} f(R z)+\lambda_{1} R\left(\frac{n z}{2}\right) \frac{f^{\prime}(R z)}{1 !}+\lambda_{2} R^{2}\left(\frac{n z}{2}\right)^{2} \frac{f^{\prime \prime}(R z)}{2 !}\right| \leq \\
& \leq R^{n}\left|\lambda_{0} z^{n}+\lambda_{1}\left(\frac{n z}{2}\right) \frac{n z^{n-1}}{1 !}+\lambda_{2}\left(\frac{n z}{2}\right)^{2} \frac{n(n-1) z^{n-2}}{2 !}\right| \max _{|z|=1}|f(z)|,
\end{aligned}
$$

for $|z| \geq 1$. The result is sharp and equality holds for $f(z) \equiv \gamma z^{n}, \gamma \neq 0$. (2).

Setting $\lambda_{1}=\lambda_{2}=0$ in inequality (14), we get in particular inequality

Remark 2. Theorem $C$ is a special case of Corollary 4, when $R=1$.

Note that if $P(z)$ is a polynomial of degree $n$ that does not vanish in $|z|<1$, then the polynomial $Q(z)=z^{n} \overline{P(1 / \bar{z})}$ has all its zeros lying in $|z| \leq 1$. Hence, if we replace $f(z)$ by $P(z)$ and $F(z)$ by $Q(z)$ in inequality (10), we get the following generalization of the result due to Aziz and Rather [13, Lemma 12].

Corollary 5. If $P(z)$ is a polynomial of degree $n$ which does not vanish in $|z|<1$, then for any complex number $\beta$ with $|\beta| \leq 1, R>r \geq 1$ and $|z|=1$ we have

$$
|B[P](R z)-\beta B[P](r z)| \leq|B[Q](R z)-\beta B[Q](r z)|,
$$

where $Q(z)=z^{n} \overline{P(1 / \bar{z})}$.

The result is sharp and equality holds for any polynomial with all its zeros on $|z|=1$. 
Further, let $M=\max _{|z|=1}|P(z)|$. Since $P(z)$ is a polynomial of degree $n$, the polynomial $P(z)+\alpha M$ for any $\alpha>1$ does not vanish in $|z|<1$; thus, applying Corollary 5 to the polynomial $P(z)+\alpha M$, we get for $|z| \geq 1$,

$$
\begin{aligned}
& |B[P](R z)-\beta B[P](r z)+\alpha(1-\beta) B[1] M| \leq \\
& \leq\left|B[Q](R z)-\beta B[Q](r z)+\bar{\alpha}\left(R^{n}-\beta r^{n}\right) B\left[E_{n}\right] M\right| .
\end{aligned}
$$

Equivalently

$$
\begin{aligned}
& \left|B[P](R z)-\beta B[P](r z)+\alpha(1-\beta) \lambda_{0} M\right| \leq \\
& \leq\left|B[Q](R z)-\beta B[Q](r z)+\bar{\alpha}\left(R^{n}-\beta r^{n}\right) B\left[E_{n}\right] M\right| .
\end{aligned}
$$

Choosing argument of $\alpha$ in the right hand side of (15) suitably, we get

$$
\begin{aligned}
\mid B[P](R z)- & \beta B[P](r z)|-| \alpha|| 1-\beta|| \lambda_{0} \mid M \leq \\
& \leq|\alpha|\left|R^{n}-\beta r^{n}\right|\left|B\left[E_{n}\right]\right| M-|B[Q](R z)-\beta B[Q](r z)| .
\end{aligned}
$$

In (16), if we make $|\alpha| \rightarrow 1$, we get for $|z| \geq 1$,

$$
\begin{gathered}
|B[P](R z)-\beta B[P](r z)|+|B[Q](R z)-\beta B[Q](r z)| \leq \\
\leq\left(\left|R^{n}-\beta r^{n}\right|\left|B\left[E_{n}\right]\right|+|1-\beta|\left|\lambda_{0}\right|\right) M .
\end{gathered}
$$

The above inequality includes many known polynomial inequalities as special cases. For example, taking $\beta=1$ and $r=1$ in (17), then dividing both sides by $(R-1)$ and making $R \rightarrow 1$, we get for any polynomial $P(z)$ of degree $n$

$$
\left|B^{\prime}[P](z)\right|+\left|B^{\prime}[Q](z)\right| \leq n\left|B\left[E_{n}\right]\right| \max _{|z|=1}|P(z)| \text { for }|z|=1,
$$

where $Q(z)=z^{n} \overline{P(1 / \bar{z})}$.

Equivalently,

$$
\begin{aligned}
& \left|\frac{d}{d z}\left\{\lambda_{0}+\lambda_{1}\left(\frac{n z}{2}\right) P^{\prime}(z)+\lambda_{2}\left(\frac{n z}{2}\right)^{2} P^{\prime \prime}(z)\right\}\right|+ \\
& +\left|\frac{d}{d z}\left\{\lambda_{0}+\lambda_{1}\left(\frac{n z}{2}\right) Q^{\prime}(z)+\lambda_{2}\left(\frac{n z}{2}\right)^{2} Q^{\prime \prime}(z)\right\}\right| \leq \\
& \leq n\left|\lambda_{0} z^{n}+\lambda_{1}\left(\frac{n z}{2}\right) \frac{n z^{n-1}}{1 !}+\lambda_{2}\left(\frac{n z}{2}\right)^{2} \frac{n(n-1) z^{n-2}}{2 !}\right| \max _{|z|=1}|P(z)| .
\end{aligned}
$$


Setting $\lambda_{0}=\lambda_{2}=0$, we have

$$
\left|\frac{d}{d z}\left(z P^{\prime}(z)\right)\right|+\left|\frac{d}{d z}\left(z Q^{\prime}(z)\right)\right| \leq n^{2} \max _{|z|=1}|P(z)|,
$$

that is,

$$
\left|P^{\prime}(z)+z P^{\prime \prime}(z)\right|+\left|Q^{\prime}(z)+z Q^{\prime \prime}(z)\right| \leq n^{2} \max _{|z|=1}|P(z)| .
$$

Combining (17) with Corollary 5, we get the following generalization of inequalities (3) and (4).

Corollary 6. If $P(z)$ is a polynomial of degree $n$ that does not vanish in $|z|<1$, then for a complex number $\beta$ with $|\beta| \leq 1, R \geq r \geq 1$ and $|z| \geq 1$, $|B[P](R z)-\beta B[P](r z)| \leq\left\{\frac{\left|R^{n}-\beta r^{n}\right|\left|B\left[E_{n}\right]\right|+\left|\lambda_{0}\right||1-\beta|}{2}\right\} \max _{|z|=1}|P(z)|$. The result is sharp and equality holds for $P(z)=e^{i \gamma}\left(\frac{z^{n}+1}{2}\right)$, where $\gamma \in \mathcal{R}$. Remark 3. Theorem $D$ is a special case of Corollary 6, if we choose $\beta=0$ and $R=1$.

Let $m=\min _{|z|=1}|P(z)|$. If the polynomial $P(z)$ has no zeros in $|z| \leq 1$, then for any $\alpha$ with $|\alpha|<1$, the polynomial $P(z)+m \alpha z^{n}$ has no zeros in $|z| \leq 1$. Hence it follows that the polynomial $Q(z)+\bar{\alpha} m$, where $Q(z)=$ $=z^{n} \overline{P(1 / \bar{z})}$ has all its zeros in $|z| \leq 1$. Thus, if in inequality (10) we take $f(z)=P(z)+m \alpha z^{n}$ and $F(z)=Q(z)+\bar{\alpha} m$, we get for $R \geq r \geq 1$ and $|z| \geq 1$

$$
\begin{aligned}
\mid B[P](R z)- & \beta B[P](r z)+\alpha m\left(R^{n}-\beta r^{n}\right) B\left[z^{n}\right] \mid \leq \\
& \leq\left|B[Q](R z)-\beta B[Q](r z)+\bar{\alpha} m(1-\beta) \lambda_{0}\right| .
\end{aligned}
$$

We choose argument of $\alpha$ in the left hand side of inequality 18 such that

$$
\begin{aligned}
& \left|B[P](R z)-\beta B[P](r z)+\alpha m\left(R^{n}-\beta r^{n}\right) B\left[z^{n}\right]\right|= \\
& =|B[P](R z)-\beta B[P](r z)|+|\alpha| m\left|R^{n}-\beta r^{n}\right| \mid B\left[z^{n} \mid,\right.
\end{aligned}
$$

so that from (18) for $|z| \geq 1$ and $R \geq r \geq 1$, we have

$$
\begin{aligned}
& \mid B[P](R z)- \beta B[P](r z)|+| \alpha|m| R^{n}-\beta r^{n}|| B\left[z^{n}\right] \mid \leq \\
& \leq|B[Q](R z)-\beta B[Q](r z)|+|\alpha| m|1-\beta|\left|\lambda_{0}\right| .
\end{aligned}
$$


If we now let $|\alpha| \rightarrow 1$, we obtain for every $\beta$ with $|\beta| \leq 1$ and $R \geq r \geq 1$,

$$
\begin{aligned}
& |B[P](R z)-\beta B[P](r z)| \leq \\
& \leq|B[Q](R z)-\beta B[Q](r z)|-m\left\{\left|R^{n}-\beta r^{n}\right|\left|B\left[z^{n}\right]\right|-|1-\beta|\left|\lambda_{0}\right|\right\}
\end{aligned}
$$

for $|z| \geq 1$. Inequality 19 in conjunction with Corollary 6 gives the following result, which is a compact generalization of results due to Aziz and Dawood [14].

Corollary 7. If $P(z)$ is a polynomial of degree $n$, which does not vanish in $|z|<1$, then for every complex number $\beta$ with $|\beta| \leq 1$ and $R \geq r \geq 1$, we have for $|z| \geq 1$,

$$
\begin{aligned}
& |B[P](R z)-\beta B[P](r z)| \leq\left\{\frac{\left|R^{n}-\beta r^{n}\right|\left|B\left[E_{n}\right]\right|+\left|\lambda_{0}\right||1-\beta|}{2}\right\} \times \\
& \quad \times \max _{|z|=1}|P(z)|-\left\{\frac{\left|R^{n}-\beta r^{n}\right|\left|B\left[E_{n}\right]\right|-\left|\lambda_{0}\right||1-\beta|}{2}\right\} \min _{|z|=1}|P(z)| .
\end{aligned}
$$

The above inequality is sharp and equality holds for $P(z) \equiv z^{n}+\gamma,|\gamma|=1$.

If we take $\beta=0$ in Corollary 7 , we get the following generalization of the result due to Shah and Liman [1].

Corollary 8. If $P(z)$ is a polynomial of degree $n$, which does not vanish in $|z|<1$, then for $R \geq 1$ and $|z| \geq 1$, we have

$$
\begin{aligned}
|B[P](R z)| \leq & \left\{\frac{R^{n}\left|B\left[E_{n}\right]\right|+\left|\lambda_{0}\right|}{2}\right\} \max _{|z|=1}|P(z)|- \\
& -\left\{\frac{R^{n}\left|B\left[E_{n}\right]\right|-\left|\lambda_{0}\right| \mid}{2}\right\} \min _{|z|=1}|P(z)| .
\end{aligned}
$$

A polynomial $P(z)$ is said to be self-inversive if $P^{*}(z)=u P(z),|u|=1$, where $P^{*}(z)=z^{n} \overline{P\left(\frac{1}{z}\right)}$. For the class of self-inversive polynomials, we have the following result which includes some known polynomial inequalities for this class as special cases.

Corollary 9. If $P(z)$ is a self-inversive polynomial of degree $n$, then for every complex number $\beta$ with $|\beta| \leq 1, R \geq r \geq 1$ and $|z| \geq 1$,

$$
|B[P](R z)-\beta B[P](r z)| \leq\left\{\frac{\left|R^{n}-\beta r^{n}\right|\left|B\left[E_{n}\right]\right|+\left|\lambda_{0}\right||1-\beta|}{2}\right\} \max _{|z|=1}|P(z)| .
$$


The result is sharp and equality holds for $P(z) \equiv z^{n}+1$.

If we take $\beta=0$ in Corollary 9 , we get the following generalized analogue of inequalities (3) and (4) for self-inversive polynomials.

Corollary 10. If $P(z)$ is a self-inversive polynomial of degree $n$, then for $R \geq 1$ and $|z| \geq 1$,

$$
|B[P](R z)| \leq\left\{\frac{R^{n}\left|B\left[E_{n}\right]\right|+\left|\lambda_{0}\right|}{2}\right\} \max _{|z|=1}|P(z)| .
$$

Equivalently

$$
\begin{aligned}
& \left|\lambda_{0} P(R z)+\lambda_{1} R\left(\frac{n z}{2}\right) \frac{P^{\prime}(R z)}{1 !}+\lambda_{2} R^{2}\left(\frac{n z}{2}\right)^{2} \frac{P^{\prime \prime}(R z)}{2 !}\right| \leq \\
& \leq\left\{\frac{R^{n}\left|\lambda_{0} z^{n}+\lambda_{1}\left(\frac{n z}{2}\right) \frac{n z^{n-1}}{1 !}+\lambda_{2}\left(\frac{n z}{2}\right)^{2} \frac{n(n-1) z^{n-2}}{2 !}\right|+\left|\lambda_{0}\right|}{2}\right\} \max _{|z|=1}|P(z)|
\end{aligned}
$$

for $|z| \geq 1$.

In Corollary 10, if we choose $\lambda_{1}=\lambda_{2}=0$, we get the result [11, Corollary 1.5]. Further, if we choose $\lambda_{0}=\lambda_{1}=0$, we get for $R>1$,

$$
\left|P^{\prime \prime}(R z)\right| \leq \frac{n(n-1) R^{n-2}|z|^{n-2}}{2 !} \max _{|z|=1}|P(z)| \text { for }|z| \geq 1,
$$

where $P(z)$ is a self-inversive polynomial.

Remark 4. The result of Rahman and Schmeisser [8, inequality 4.5.6] is a special case of Theorem 2, if we take $F(z)=z^{n}$.

Let $P(z)$ be a polynomial of degree $n$ having no zeros in $|z|<1$, then $Q(z)=z^{n} \overline{P\left(\frac{1}{\bar{z}}\right)}$ is a polynomial of degree $n$, having all zeros in $|z| \leq 1$ and $|P(z)| \leq|Q(z)|$ for $|z|=1$. Therefore by Theorem 2 , for $|z| \geq 1$,

$$
\left|\lambda n^{2} P(z)-z P^{\prime}(z)-z^{2} P^{\prime \prime}(z)\right| \leq\left|\lambda n^{2} Q(z)-z Q^{\prime}(z)-z^{2} Q^{\prime \prime}(z)\right|,
$$

where $\lambda$ belongs to the set

$$
\triangle:=\left\{w=\rho+i \sigma: 0 \leq \sigma^{2} \leq \frac{n}{n-1}\left(\frac{n+1}{4 n}-\rho\right), \rho \leq \frac{n+1}{4 n}\right\} .
$$


Proceeding similarly as in Corollary 6 and Corollary 10, we get inequalities (14.5.6) and (14.5.7) of 8$]$ as special cases.

5. Lemmas. To prove the theorems we need the following lemmas. The first lemma is due to Aziz and Zargar [15] (see also Govil et. al. [12]).

Lemma 1. If $f(z):=\sum_{\nu=0}^{n} a_{\nu} z^{\nu}$ is a polynomial of degree $n$ having all its zeros in $|z| \leq k$ where $k \geq 0$, then for every $R \geq r$ and $r R \geq k^{2}$,

$$
|f(R z)| \geq\left(\frac{R+k}{r+k}\right)^{n}|f(r z)| \text { for }|z|=1 .
$$

The next Lemma which we need follows from [14, Corollary 18.3].

Lemma 2. If $f(z)$ is a polynomial of degree $n$ having all its zeros in $|z| \leq 1$, then all zeros of $B[P](z)$ also lie in $|z| \leq 1$.

Lemma 3. For every $a>0$ and $w \in \mathcal{C}$ the following equivalence hold:

$$
|\operatorname{Re}(w)| \leq a
$$

if and only if

$$
w^{2} \in \triangle:=\left\{x+i y \in \mathcal{C}: x, y \in \mathcal{R}, x \leq a^{2}-\frac{y^{2}}{4 a^{2}}\right\} .
$$

Proof. We note that Lemma 3 simply follows, if we consider the region $\{w \in \mathcal{C}:|\operatorname{Re}(w)| \leq a\}$ as the union of vertical lines contained in it and then look at the images of these lines under the square function. However, we give here an analytic proof as well for the readers' convenience. For this suppose that $|\operatorname{Re}(w)| \leq a$. If $w=u+i v$, then $|\operatorname{Re}(w)|=|u| \leq a$. Since $w^{2}=x+i y, x=u^{2}-v^{2}$ and $y=2 u v$. This gives $x \leq a^{2}-v^{2}$ and $y^{2} \leq 4 a^{2} v^{2}$ and simplifying we obtain

$$
x \leq a^{2}-\frac{y^{2}}{4 a^{2}} .
$$

This shows that $w^{2} \in \triangle$. Conversely if $w^{2} \in \triangle$, then

$$
x \leq a^{2}-\frac{y^{2}}{4 a^{2}},
$$

so that on substituting for $x$ and $y$, we get

$$
|u| \leq a .
$$


This shows that $|\operatorname{Re}(w)| \leq a$ and Lemma 3 is proved.

Lemma 4. Let $n>1$ be a positive integer and $\lambda \in \mathcal{C}$. Assume that

$$
T(z)=z^{2}+\frac{n}{2(n-1)} z-\frac{\lambda n^{3}}{4(n-1)}=0 .
$$

Then

$$
\lambda \in \triangle:=\left\{\rho+i \sigma \in \mathcal{C}: \rho, \sigma \in \mathcal{R}, \rho \leq \frac{n+1}{4 n}-\frac{n-1}{n} \sigma^{2}\right\},
$$

if and only if

$$
-\frac{n^{2}+n}{4(n-1)} \leq \operatorname{Re}(z) \leq \frac{n}{4}
$$

Proof. We have

$$
T(z)=z^{2}+\frac{n}{2(n-1)} z-\frac{\lambda n^{3}}{4(n-1)}=0 .
$$

By completing the square, we get

$$
\left(z+\frac{n}{4(n-1)}\right)^{2}=\frac{n^{2}}{4(n-1)}\left(\frac{1}{4(n-1)}+\lambda n\right) .
$$

We take $w=z+\frac{n}{4(n-1)}$ and $a=\frac{n^{2}}{4(n-1)}$, so that $|\operatorname{Re}(w)| \leq a$ and by Lemma 3

$$
w^{2} \in\left\{x+i y \in \mathcal{C}, x, y \in \mathcal{R}, x \leq a^{2}-\frac{y^{2}}{4 a^{2}}\right\} .
$$

Therefore by (20) we have

$$
\frac{n^{2}}{4(n-1)}\left(\frac{1}{4(n-1)}+\lambda n\right) \in\left\{x+i y \in \mathcal{C}, x, y \in \mathcal{R}, x \leq a^{2}-\frac{y^{2}}{4 a^{2}}\right\} \text {. }
$$

But

$$
\begin{aligned}
\frac{n^{2}}{4(n-1)}\left(\frac{1}{4(n-1)}+\lambda n\right) & =a\left(\frac{a}{n^{2}}+\lambda n\right)= \\
& =a\left(\frac{a}{n^{2}}+(\rho+i \sigma) n\right)= \\
& =a\left(\frac{a}{n^{2}}+\rho n\right)+i a \sigma n
\end{aligned}
$$


Setting

$$
x=a\left(\frac{a}{n^{2}}+\rho n\right) \quad \text { and } \quad y=a \sigma n,
$$

so that

$$
w^{2}=\frac{n^{2}}{4(n-1)}\left(\frac{1}{4(n-1)}+\lambda n\right)=x+i y .
$$

By this substitution we have

$$
x \leq a^{2}-\frac{y^{2}}{4 a^{2}}
$$

implies

$$
\rho \leq \frac{n+1}{4 n}-\frac{n-1}{n} \sigma^{2} .
$$

Also, it can be easily verified that $|R e(w)| \leq a$ and hence

$$
-\frac{n^{2}+n}{4(n-1)} \leq \operatorname{Re}(z) \leq \frac{n}{4}
$$

This proves Lemma 4 also.

\section{Proofs of the Theorems.}

Proof of Theorem 1. We first suppose that $R=r$. By hypothesis $|f(z)| \leq|F(z)|$ for $|z|=1$, therefore, for every complex number $\delta$ with $|\delta|>1$,we have $|f(z)|<|\delta F(z)|$ for $|z|=1$. Further, all zeros of $F(z)$ lie in $|z| \leq 1$; it follows from Rouché's theorem [16, p.304] that all zeros of a polynomial $g(z):=f(z)-\delta F(z)$, of degree $n$ also lie in $|z| \leq 1$. Therefore by Lemma 2 , all zeros of

$$
\begin{aligned}
& B[g](z)=B[f-\delta F](z)= \\
& =\lambda_{0}(f-\delta F)(z)+\lambda_{1} \frac{n z}{2}\left\{f^{\prime}-\delta F^{\prime}\right\}(z)+\lambda_{2}\left(\frac{n z}{2}\right)^{2}\left\{\frac{f^{\prime \prime}(z)-\delta F^{\prime \prime}(z)}{2 !}\right\}= \\
& =\left\{\lambda_{0} f(z)+\lambda_{1} \frac{m z}{2} f^{\prime}(z)+\lambda_{2}\left(\frac{m z}{2}\right)^{2} \frac{f^{\prime \prime}(z)}{2 !}\right\}-\delta\left\{\lambda_{0} F(z)+\lambda_{1} \frac{n z}{2} F^{\prime}(z)+\right. \\
& \left.+\lambda_{2}\left(\frac{n z}{2}\right)^{2} \frac{F^{\prime \prime}(z)}{2 !}\right\}+\lambda_{1}\left(\frac{n-m}{2}\right) z f^{\prime}(z)+\lambda_{2}\left(\frac{n^{2}-m^{2}}{8}\right) z^{2} f^{\prime \prime}(z)= \\
& =B[f](z)-\delta B[F](z)+\lambda_{1}\left(\frac{n-m}{2}\right) z f^{\prime}(z)+\lambda_{2}\left(\frac{n^{2}-m^{2}}{8}\right) z^{2} f^{\prime \prime}(z)
\end{aligned}
$$


are in $|z| \leq 1$.

This gives

$$
\left|B[f](z)+\lambda_{1}\left(\frac{n-m}{2}\right) z f^{\prime}(z)+\lambda_{2}\left(\frac{n^{2}-m^{2}}{8}\right) z^{2} f^{\prime \prime}(z)\right| \leq|B[F](z)|
$$

for $|z|>1$, where $\lambda_{0}, \lambda_{1}$ and $\lambda_{2}$ are such that $u(z):=\lambda_{0}+C(n, 1) \lambda_{1} z+$ $+C(n, 2) \lambda_{2} z^{2}$ has all zeros in $\operatorname{Re}(z) \leq \frac{n}{4}$, and $v(z):=\lambda_{0}+C(m, 1) \lambda_{1} z+$ $+C(m, 2) \lambda_{2} z^{2}$ has all zeros in $\operatorname{Re}(z) \leq \frac{m}{4}$. If inequality 22 is not true, then there is a point $z=z_{0}$ with $\left|z_{0}\right|>1$, such that

$$
\left|B[f]\left(z_{0}\right)+\lambda_{1}\left(\frac{n-m}{2}\right) z_{0} f^{\prime}\left(z_{0}\right)+\lambda_{2}\left(\frac{n^{2}-m^{2}}{8}\right) z_{0}^{2} f^{\prime \prime}\left(z_{0}\right)\right|>\left|B[F]\left(z_{0}\right)\right| .
$$

Since all zeros of $F(z)$ lie in $|z| \leq 1$, Lemma 2 shows that $B[F]\left(z_{0}\right) \neq 0$ for any $z_{0}$ with $\left|z_{0}\right|>1$. Hence we can choose

$$
\delta=\frac{\left|B[f]\left(z_{0}\right)+\lambda_{1}\left(\frac{n-m}{2}\right) z_{0} f^{\prime}\left(z_{0}\right)+\lambda_{2}\left(\frac{n^{2}-m^{2}}{8}\right) z_{0}^{2} f^{\prime \prime}\left(z_{0}\right)\right|}{\left|B[F]\left(z_{0}\right)\right|}
$$

so that $\delta$ is a well defined complex number such that $|\delta|>1$ and with such choice of $\delta$ we get from (21) $B[g]\left(z_{0}\right)=0$, for $\left|z_{0}\right|>1$. This shows that all zeros of $B[g](z)$ lie in $|z|>1$, which is a contradiction and hence from 22) after substituting for $z=R e^{i \theta}, R>1$ and noting that $(f(R z))^{\prime}=$ $=R f^{\prime}(R z)$, we have for $R>1$,

$$
\left|B[f](R z)+\lambda_{1}\left(\frac{n-m}{2}\right) z(f(R z))^{\prime}+\lambda_{2}\left(\frac{n^{2}-m^{2}}{8}\right) z^{2}(f(R z))^{\prime \prime}\right| \leq|B[F](R z)| .
$$

By using the argument of continuity, we show that the theorem is true for $R=r \geq 1$. We now assume that $R>r$. Since $g(z)$ has all zeros in $|z| \leq 1, g\left(R e^{i \theta}\right) \neq 0$ for every $R>r \geq 1,0 \leq \theta<2 \pi$. Hence we have

$$
\left|g\left(R e^{i \theta}\right)\right|>\left(\frac{r+1}{R+1}\right)^{n}\left|g\left(R e^{i \theta}\right)\right|
$$

Also, if we apply Lemma 1 with $k=1$ to the polynomial $g(z):=f(z)-$ $-\delta F(z)$, we get for $R \geq r \geq 1$,

$$
\left|g\left(R e^{i \theta}\right)\right| \geq\left(\frac{R+1}{r+1}\right)^{n}\left|g\left(r e^{i \theta}\right)\right|, \quad 0 \leq \theta<2 \pi .
$$


Combining (24) with (23) gives for $R>r \geq 1$,

$$
|g(r z)|<|g(R z)|, \quad|z|=1 .
$$

If $\beta$ is any complex number with $|\beta| \leq 1$, then it follows from $(25)$ that $|\beta g(r z)|<|g(R z)|$ for $|z|=1$ and $R>r \geq 1$. As all zeros of $g(R z)$ lie in $|z| \leq \frac{1}{R}<1$, therefore again by using Rouché's theorem, we how that the polynomial

$$
h(z):=g(R z)-\beta g(r z)=f(R z)-\beta f(r z)-\delta(F(R z)-\beta F(r z))
$$

has all its zeros in $|z|<1$, for every complex number $\delta$ with $|\delta|>1$ and $R>r \geq 1$, therefore by Lemma 2 , all zeros of $B[h](z)$ lie in $|z|<1$. Since $f(z)$ and $F(z)$ are polynomials of degree $m$ and $n(m \leq n)$ respectively, $h(z)$ is a polynomial of degree $n$ and hence

$$
\begin{aligned}
& B[h](z)=B[f(R z)-\beta f(r z)-\delta(F(R z)-\beta F(r z))]= \\
& \quad=\lambda_{0}[f(R z)-\beta f(r z)-\delta(F(R z)-\beta F(r z))]+ \\
& \quad+\lambda_{1} \frac{n z}{2}\left[(f(R z))^{\prime}-\beta(f(r z))^{\prime}-\delta\left\{(F(R z))^{\prime}-\beta(F(r z))^{\prime}\right\}\right]+ \\
& \quad+\frac{\lambda_{2}}{2 !}\left(\frac{n z}{2}\right)^{2}\left[(f(R z))^{\prime \prime}-\beta(f(r z))^{\prime \prime}-\delta\left\{(F(R z))^{\prime \prime}-\beta(F(r z))^{\prime \prime}\right\}\right]= \\
& \quad=B[f](R z)-\beta B[f](r z)+\lambda_{1}\left(\frac{n-m}{2}\right) z\left[(f(R z))^{\prime}-\beta(f(r z))^{\prime}\right]+ \\
& \quad+\lambda_{2} \frac{n^{2}-m^{2}}{8} z^{2}\left[(f(R z))^{\prime \prime}-\beta(f(r z))^{\prime \prime}\right]-\delta\{B[F](R z)-\beta B[F](r z)\}
\end{aligned}
$$

has all zeros in $|z|<1$.

From this we conclude as above that

$$
\begin{aligned}
& \mid B[f](R z)-\beta B[f](r z)+\lambda_{1}\left(\frac{n-m}{2}\right) z\left\{(f(R z))^{\prime}-\beta(f(r z))^{\prime}\right\}+ \\
& +\lambda_{2}\left(\frac{n^{2}-m^{2}}{8}\right)^{2}\left[(f(R z))^{\prime \prime}-\beta(f(r z))^{\prime \prime}\right]|\leq| B[F](R z)-\beta B[F](r z) \mid
\end{aligned}
$$

for every $R>r \geq 1$.

Now in order to complete the proof, we need only to show that for $|z|>1$, the inequality $(10)$ becomes a strict inequality and this is done as follows. Note that, $B[f](R z)-\beta B[f](r z)+\lambda_{1}\left(\frac{n-m}{2}\right) z\left\{(f(R z))^{\prime}-\beta\left(f(r z)^{\prime}\right)\right\}+$ 
$+\lambda_{2}\left(\frac{n^{2}-m^{2}}{8}\right)^{2}\left[(f(R z))^{\prime \prime}-\beta(f(r z))^{\prime \prime}\right]$ and $B[F](R z)-\beta B[F](r z)$ are two polynomials, where degree of $B[f](R z)-\beta B[f](r z)+\lambda_{1}\left(\frac{n-m}{2}\right) \times$ $\times z\left\{(f(R z))^{\prime}-\beta\left(f(r z)^{\prime}\right)\right\}+\lambda_{2}\left(\frac{n^{2}-m^{2}}{8}\right)^{2}\left[(f(R z))^{\prime \prime}-\beta(f(r z))^{\prime \prime}\right]$ does not exceed that of $B[F](R z)-\beta B[F](r z)$, and the polynomial $B[F](R z)-\beta B[F](r z)$ has all its zeros in $|z| \leq 1$. Further, by $(10)$ we have for $|z|=1, \mid B[f](R z)-\beta B[f](r z)+\lambda_{1}\left(\frac{n-m}{2}\right) z\left\{(f(R z))^{\prime}-\beta\left(f(r z)^{\prime}\right)\right\}+$ $+\lambda_{2}\left(\frac{n^{2}-m^{2}}{8}\right)^{2}\left[(f(R z))^{\prime \prime}-\beta(f(r z))^{\prime \prime}\right]|\leq| B[F](R z)-\beta B[F](r z) \mid$.

Therefore, by applying Theorem B we get

$$
\begin{aligned}
& \mid B[f](R z)-\beta B[f](r z)+\lambda_{1}\left(\frac{n-m}{2}\right) z\left\{(f(R z))^{\prime}-\beta\left(f(r z)^{\prime}\right)\right\}+ \\
& +\lambda_{2}\left(\frac{n^{2}-m^{2}}{8}\right)^{2}\left\{(f(R z))^{\prime \prime}-\beta(f(r z))^{\prime \prime}\right\}|<| B[F](R z)-\beta B[F](r z) \mid
\end{aligned}
$$

for $|z|>1$. That is 10 becomes a strict inequality for $|z|>1$ and Theorem 1 is thus completely proved.

Proof of Theorem 2. We choose $\lambda_{0}=n^{2} \lambda, \lambda_{1}=\frac{-2}{n}, \lambda_{2}=\frac{-8}{n^{2}}$, where

$\lambda$ is such that

$$
\lambda \in \triangle:=\left\{w=\rho+i \sigma: 0 \leq \sigma^{2} \leq \frac{n}{n-1}\left(\frac{n+1}{4 n}-\rho\right), \rho \leq \frac{n+1}{4 n}\right\} .
$$

By this choice of $\lambda$, we get by using Lemma 4,

$$
\begin{aligned}
& \lambda_{0}+C(n, 1) \lambda_{1} z+C(n, 2) \lambda_{2} z^{2}= \\
& =-\frac{4(n-1)}{n}\left\{z^{2}+\frac{n}{2(n-1)} z-\frac{\lambda n^{3}}{4(n-1)}\right\}=0
\end{aligned}
$$

for $\operatorname{Re}(z) \leq \frac{n}{4}$.

Thus for this value of $\lambda \in \triangle, B$ defined by $(8)$ is a $\mathcal{B}_{n}$-operator. Hence by Theorem 1 , with $m=n, \beta=0$ and $R=1$, we have,

$$
|B[f](z)| \leq|B[F](z)| \quad \text { for } \quad|z| \geq 1
$$


This in particular gives for $|z| \geq 1$,

$$
\begin{aligned}
\mid \lambda_{0} f(z)+\lambda_{1}\left(\frac{n z}{2}\right) \frac{f^{\prime}(z)}{1 !}+ & \lambda_{2}\left(\frac{n z}{2}\right)^{2} \frac{f^{\prime \prime}(z)}{2 !} \mid \leq \\
& \leq\left|\lambda_{0} F(z)+\lambda_{1}\left(\frac{n z}{2}\right) \frac{F^{\prime}(z)}{1 !}+\lambda_{2}\left(\frac{n z}{2}\right)^{2} \frac{F^{\prime \prime}(z)}{2 !}\right| .
\end{aligned}
$$

Substituting value of $\lambda_{0}, \lambda_{1}$ and $\lambda_{2}$ in (26) we get for $\lambda \in \triangle$ and $|z| \geq 1$

$$
\left|\lambda n^{2} f(z)-z f^{\prime}(z)-z^{2} f^{\prime \prime}(z)\right| \leq\left|\lambda n^{2} F(z)-z F^{\prime}(z)-z^{2} F^{\prime \prime}(z)\right| .
$$

This completes the proof of Theorem 2 .

Acknowledgment. Authors are grateful to the referees for the careful reading and useful suggestions.

\section{References}

[1] Bernstein S. Sur la limitation des dérivées des polynomes. C. R. Acad. Sci. Paris., 1930, vol. 190, pp. 338-340.

[2] Polya G. and Szego G. Problems and Theorems in Analysis. New York: Springer Verlag, 1972.

[3] Lax P. D. Proof of a conjecture of P. Erdös on the derivative of a polynomial. Bull. Amer. Math. Soc., 1944, vol. 50, pp. 509-513.

[4] Ankeny N. C. and Rivlin T. J. On a Theorem of S. Bernstein. Pacific. J. Math., 1955, vol. 5, pp. 849-852.

[5] Frappier C., Rahman Q. I. and Ruscheweyh St. New inequalities for polynomials. Trans. Amer. Math. Soc., 1985, vol. 88, pp. 69-99.

[6] Bernstein S. Lecons Sur les propriétés extrémales et la meilleure approximation des function analytiques dúne variable réele. GauthierVillars. Paris, 1926.

[7] Govil N. K. On the derivative of a polynomial. Proc. Amer. Math. Soc., 1973, vol. 41, pp. 543-546.

[8] Rahman Q. I. and Schmeisser G. Analytic Theory of Polynomials. Oxford University Press, New York, 2002.

[9] Rahman Q. I. Functions of exponential type, Trans. Amer. Math. Soc., 1969, vol. 135, pp. 295-309.

[10] Marden M. Geometry of polynomials, 2nd ed. Mathematical Surveys No. 3, Amer. Math. Soc., Providence, RI. 1966. 
[11] Shah W. M. and Liman A. An operator preserving inequalities between polynomials. J. Inequal. Pure Appl. Math., 2008, vol. 9, pp. 1-12.

[12] Govil N. K., Liman N. K. and Shah W. M. Some inequalities concerning derivative and maximum modulus of polynomials. Aust. J. Math. Anal. Appl., 2011, vol. 8, pp. 1-8.

[13] Aziz A. and Rather N. A. Some compact generalizations of Zygmund type inequalities for polynomials. Nonlinear Studies., 1999, vol. 6, pp. 241-255.

[14] Aziz A. and Dawood Q. M. Inequalities for a polynomial and its derivative. J. Approx. Theory, 1988, vol. 53, pp. 155-162.

[15] Aziz A. and Zargar B. A. Inequalities for a polynomial and its derivative. Math. Inequal. Appl. 1998, vol. 4, pp. 543-550.

[16] Ponnusamy S. Foundations of Complex Analysis, 2nd ed. Narosa Publishing House Pvt. Ltd, 2005.

Received July 11, 2016.

In revised form, October 10, 2016.

Accepted October 10, 2016.

National Institute of Technology

Srinagar,190006 Kashmir, India

E-mail: abliman22@yahoo.com ${ }^{3}$, shahlw@yahoo.co.in ${ }^{1}$

Jammu and Kashmir Institute of Mathematical Sciences

Srinagar,190008 Kashmir, India

E-mail: wmshah@rediffmail.com² 